\title{
Assessing spatial heterogeneity of multidrug-resistant tuberculosis in a high-burden country
}

\author{
Helen E. Jenkins ${ }^{1,2}$, Valeriu Plesca ${ }^{3}$, Anisoara Ciobanu ${ }^{3}$, Valeriu Crudu ${ }^{4}$, \\ Irina Galusca ${ }^{3}$, Viorel Soltan ${ }^{4}$, Aliona Serbulenco ${ }^{5}$, Matteo Zignol ${ }^{6}$, Andrei Dadu 7 , \\ Masoud Dara ${ }^{7}$ and Ted Cohen ${ }^{1,8}$
}

Affiliations: 'Brigham and Women's Hospital, Boston, ${ }^{2}$ Harvard Medical School, Boston, and ${ }^{8}$ Harvard School of Public Health, Boston, MA, USA. ${ }^{3}$ National Centre of Health Management, Chisinau, ${ }^{4}$ Center for Health Policies and Studies, Chisinau, and ${ }^{5}$ Ministry of Health, Chisinau, Republic of Moldova. ${ }^{6}$ Stop TB Dept, World Health Organization, Geneva, Switzerland. ${ }^{7}$ World Health Organization, Regional Office for Europe, Copenhagen, Denmark.

Correspondence: H.E. Jenkins, Brigham and Women's Hospital, 641 Huntington Avenue, Boston, MA 02115, USA. E-mail: jenkins.helendgmail.com

ABSTRACT Multidrug-resistant tuberculosis (MDR-TB) is a major concern in countries of the former Soviet Union. The reported risk of resistance among tuberculosis (TB) cases in the Republic of Moldova is among the highest in the world. We aimed to produce high-resolution spatial maps of MDR-TB risk and burden in this setting.

We analysed national TB surveillance data collected between 2007 and 2010 in Moldova. High drug susceptibility testing coverage and detailed location data permitted identification of subregional areas of higher MDR-TB risk. We investigated whether the distribution of cases with MDR-TB risk factors could explain this observed spatial variation in MDR-TB.

3447 MDR-TB cases were notified during this period; $24 \%$ of new and $62 \%$ of previously treated patients had MDR-TB. Nationally, the estimated annual MDR-TB incidence was 54 cases per 100000 persons and $>1000$ cases per 100000 persons within penitentiaries. We identified substantial geographical variation in MDR-TB burden and hotspots of MDR-TB. Locations with a higher percentage of previously incarcerated TB cases were at greater risk of being MDR-TB hotspots.

Spatial analyses revealed striking geographical heterogeneity of MDR-TB. Methods to identify locations of high MDR-TB risk and burden should allow for better resource allocation and more appropriate targeting of studies to understand local mechanisms driving resistance.

@ERSpublications

Spatial analyses reveals striking geographical heterogeneity of MDR-TB http://ow.ly/oO5Xx

This article has supplementary material available from www.erj.ersjournals.com

Received: July 182012 | Accepted after revision: Sept 192012 | First published online: Oct 252012

Support statement: This work was supported by Award Number U54GM088558 from the National Institute of General Medical Sciences.

Conflict of interest: Disclosures can be found alongside the online version of this article at www.erj.ersjournals.com 


\section{Introduction}

Intensification of global tuberculosis $(\mathrm{TB})$ control efforts has contributed to declines in estimated incidence and mortality [1]. However, drug-resistant TB threatens recent successes, which are built upon the use of standardised drug regimens [2]. In 2009-2010, the highest ever levels of multidrug-resistant TB (MDR-TB) (i.e. TB that is resistant to at least isoniazid and rifampicin and does not respond to first-line combination therapy [3]) were reported [4,5]. MDR-TB control is hampered by the challenges in both detecting resistant disease through drug susceptibility testing (DST) and access to quality assured treatment [6]. In 2010, of the 290000 estimated MDR-TB cases among notified pulmonary TB cases globally, only 16\% were diagnosed and initiated on appropriate treatment [1].

Countries of the former Soviet Union (FSU) have reported percentages of TB cases with MDR-TB several times higher than other countries $[1,4,7,8]$. The Republic of Moldova is a small country (area $33846 \mathrm{~km}^{2}$, population 4 million in 2010) that gained independence from the Soviet Union in 1991. Challenging economic conditions have resulted in substantial emigration, mainly to Europe, and currently 800000 Moldovans are estimated to be abroad [9]. Like many areas of the FSU, Moldova has a serious MDR-TB problem: a 2006 nationwide TB drug-resistance survey found that $19.4 \%$ and $50.8 \%$ of new and previously treated TB cases, respectively, had MDR-TB $[8,10]$. Consequently, substantial investments were made in Moldova to improve TB diagnosis, surveillance and treatment [7, 11]. Notable achievements include the expansion of DST coverage, which is mandated for all culture-positive cases (an exceptionally rare policy in high TB prevalence countries), and the development of an online data collection system that incorporates laboratory, clinical and demographic information.

Identifying where MDR-TB is geographically concentrated should help to inform the rational allocation of resources and allow for targeted studies that aim to identify local drivers of emergence and spread of resistance. Here, we use programmatic data collected between 2007 and 2010 in Moldova to describe the risk and burden of MDR-TB both nationwide and locally. We also produce detailed maps to illustrate spatial variation in MDR-TB. Finally, we identify host-level risk factors of multidrug resistance among TB cases and investigate whether the spatial distribution of such risk factors can explain the observed geographical variation in MDR-TB.

\section{Methods}

Data sources

We used the Moldovan TB database of all notified TB cases diagnosed nationwide between January 2007 and December 2010 (23 152 cases). TB cases are diagnosed by sputum smear microscopy, culture and/or abnormal radiography in the presence of symptoms. Detailed demographic and residential location data on each notified case are collected at the time of TB diagnosis and entered into the centralised online database. Laboratory results are added to this database when available and all data are verified by staff at the National Centre of Health Management. Data are also included from Transnistria, a semi-autonomous region in eastern Moldova, which operates largely independently but contributes cases to the national TB database (fig. 1).

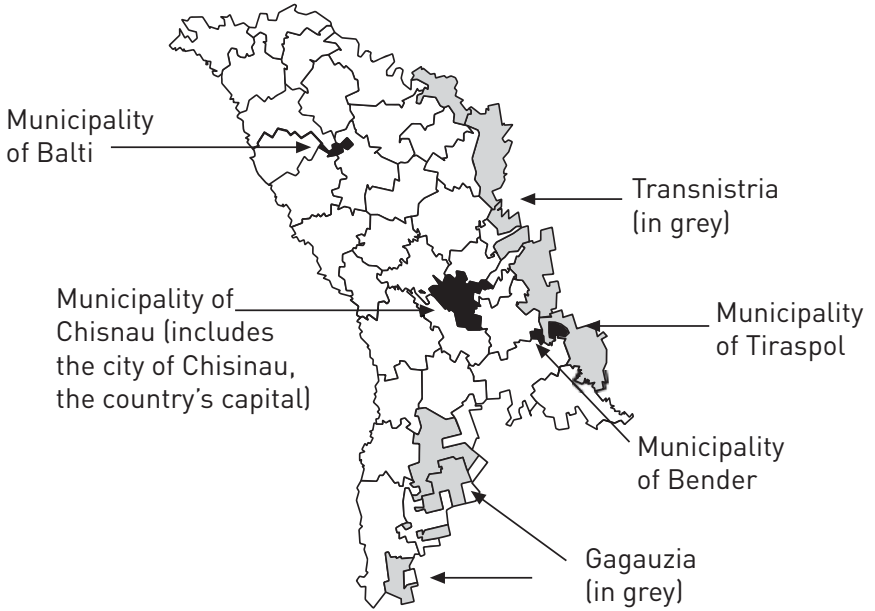

FIGURE 1 Map of the Republic of Moldova. Borders of the administrative regions are shown that highlight the four municipalities (in black) and two semi-autonomous regions (in grey). Note that Moldova is largely rural and the four municipalities shown are the main urban and highest population density areas. The total area of the country is $\sim 34000 \mathrm{~km}^{2}$. 
Moldova has four laboratories performing culture and DST (including one national reference laboratory) that have passed external quality assurance conducted by the Supranational Reference Laboratory Network in Borstel, Germany [8]. DST was performed on solid culture media using the absolute concentration method [10].

We obtained population estimates from the most recent census in 2004 [12]. Moldova is divided, administratively, into 44 regions ("rayons") and 1681 "localities" (villages or sections within a city). Thus, residential location data enabled us to pinpoint a case to a locality within a rayon.

\section{Statistical analysis}

Assessing diagnostic tools and MDR-TB burden

We calculated the percentage of notified pulmonary TB cases receiving smear microscopy, mycobacterial culture, and DST by year. MDR-TB cases were those with resistance to at least isoniazid and rifampicin. Non-MDR-TB cases were those who received DST and were susceptible to isoniazid and/or rifampicin. Using data from TB cases with sufficient DST to be classified as either MDR-TB or non-MDR-TB, we calculated the percentage of cases with MDR-TB separately for new TB patients (i.e. those who have either previously received treatment for $<1$ month in total or never) and previously treated TB patients (i.e. those who have previously received $\geqslant 1$ month of TB treatment) [13].

As TB cases without a culture-positive sample did not receive DST, MDR-TB notifications are likely to underreport the total MDR-TB burden. We used two different approaches for estimating the actual burden of MDR-TB. First, as a conservative lower bound, we estimated MDR-TB incidence assuming that only notified TB cases with a positive smear and/or culture could have had MDR-TB. Secondly, we estimated the MDR-TB incidence assuming that all notified cases (i.e. cases with or without microbiological confirmation) could have had MDR-TB. Both of these approaches assume that cases without DST results were as likely to have had MDR-TB as those who were tested, as DST was carried out for nearly all culturepositive TB cases (and not targeted only at those presumed more likely to have MDR-TB).

We report the use of diagnostic tools, incidence of notified new and previously treated MDR-TB cases, and percentage of TB cases with MDR-TB by rayon. When estimating MDR-TB incidence stratified by rayon, we used rayon-specific estimated percentages of MDR-TB, stratified by new or previously treated status, and rayon-specific notified TB incidences. We repeated these analyses for patients diagnosed within the penitentiary system.

\section{Individual-level factors associated with MDR-TB among TB cases}

We constructed two logistic regression models to identify individual-level (i.e. host-specific) variables that were significantly associated with the odds of having MDR-TB among new and previously treated TB cases. Factors that increase an individual's odds of having MDR-TB among these two groups may differ as resistance among new cases reflects MDR-TB transmission whereas resistance among previously treated cases may result from transmitted resistance or resistance acquired during prior exposure to TB drugs.

A full model was constructed including all potential explanatory variables to obtain fully adjusted odds ratios. Some cases were missing data on some variables (table S1) and hence inclusion of these variables would reduce the sample size used in the model. Therefore, we examined only those variables for which $<10 \%$ were missing data. A backwards elimination method was used to identify variables that were statistically significantly associated with MDR-TB diagnosis. In addition, any nonstatistically significant variable which, on removal, altered other parameter estimates substantially $(>10 \%)$ remained in the model to ensure full adjustment for confounding (methods in online supplementary material).

\section{Geographical heterogeneity of the incidence of MDR-TB}

In addition to the analysis of MDR-TB burden by rayon described above, we constructed maps of MDR-TB burden. The percentage of TB cases with MDR-TB and notified and estimated MDR-TB incidences were aggregated by locality and plotted using latitude and longitude co-ordinates. As these provided estimates at specific locations, we used inverse distance weighting [14] to produce maps that allow visualisation of smoothed estimates of incidence across the entire country. This method divides the country into cells of a pre-specified area and estimates the value of interest (i.e. MDR-TB incidence or percentage with MDR-TB) in each cell. This is done by calculating an average of the values from the nearest $\mathrm{N}$ points, inversely weighting them by the distance of each point from the cell so that nearby points have a large influence and further points have less (for technical details, see the online supplementary material). To test for local spatial clustering and outliers of high MDR-TB risk, we used Anselin Local Moran's I (online supplementary material) [15]. 
We then assessed whether a concentration of cases with factors that increased the odds of having MDR-TB (identified by the methods described above) could explain the increased MDR-TB burden observed in some localities. We used two logistic regression models (for new and previously treated TB cases) to model the proportion of TB cases with DST that had MDR-TB in each locality. For each individual-level factor identified above, we created an explanatory variable for the proportion of all TB cases in the locality that had that factor. All variables were log transformed before inclusion in the model [16]. We assessed the predictive ability of the model using receiver operating characteristic (ROC) curves where an area under the curve (AUC) value represents the predictive probability of the model (AUC 0.5, none; AUC 0.5-0.7, poor; AUC 0.7-0.9, reasonable; AUC $>0.9$, very good) (online supplementary material) [17].

We did not make any adjustment for potential spatial variation in TB case detection rates. We made the assumptions that 1) the percentages of undetected TB cases that had MDR-TB were equivalent to those among detected $\mathrm{TB}$ cases and 2) there was no substantial spatial variation in $\mathrm{TB}$ case detection rates. Analyses were carried out using SAS statistical software, version 9.2 (SAS Institute Inc., Cary, NC, USA) and ArcMap version 10.0 (Esri; Redlands, CA, USA).

\section{Results}

From 2007 to 2010, there were 23152 notified TB cases in Moldova (table S2). 98\% of notified pulmonary $\mathrm{TB}$ cases had sputum specimens examined by microscopy and $92 \%$ received culture examination (fig. S1). Approximately half of all notified pulmonary TB cases were culture-positive and $94 \%$ of notified culturepositive TB cases received DST. Generally, the use of diagnostic tools in the penitentiary system was comparable with the civilian sector. However, in Transnistria, only 59\% of pulmonary TB cases received culture and only $73 \%$ of culture-positive cases received DST (table S2).

Between 2007 and 2010, there were 3447 notified MDR-TB cases accounting for 38\% of TB cases that received DST for MDR-TB (23.5\% of new cases and $61.5 \%$ of previously treated cases had MDR-TB (table 1)). Among new cases, younger cases were more likely to have MDR-TB (fig. 2). While the annual rate of notified MDR-TB per 100000 people was 20.9 cases, this statistic underreports the total burden as many TB cases were culture negative and thus unable to receive a DST. Therefore, we estimated an annual MDR-TB incidence of as much as 54 cases per 100000 people, dependent on the method used to adjust for underreporting (table 1).

Within the penitentiary system, there were $1689 \mathrm{~TB}$ cases ( $7.3 \%$ of all notified TB cases in Moldova). In prisons outside Transnistria, 37\% of new and $83 \%$ of previously treated cases had MDR-TB (table 1). In Transnistrian prisons, more than three-quarters of TB cases had MDR-TB. The estimated MDR-TB incidence in the entire penitentiary system may be $>1000$ annual cases per 100000 persons; $>20$ times that estimated for the rest of Moldova.

\section{Individual-level factors associated with MDR-TB among TB cases}

We identified several characteristics for new and previously treated TB cases that were independently associated with having MDR-TB (table 2). Several potential factors, such as sex, smear status, education level and household size, were not independently associated with MDR-TB in new or previously treated TB cases (table S10).

\section{Geographical heterogeneity in MDR-TB incidence}

Both the percentage of tested TB cases with MDR-TB and the estimated MDR-TB incidence varied substantially between rayons (fig. 2). Among new TB cases, the percentage with MDR-TB ranged from $2.9 \%$ to $41.4 \%$ and among previously treated cases from $41.0 \%$ to $78.9 \%$. The ranges in the annual notification and estimated annual incidence of MDR-TB were similarly wide (fig. S2).

Mapping of notified MDR-TB cases by locality revealed marked heterogeneity even within single rayons (figs 3 and 4). Spatial clustering analysis highlighted several areas with statistically significant clustering of high MDR-TB risk and localities that had substantially higher MDR-TB risk than those around them (fig. $3 \mathrm{c}$ and d). Our locality-level regression showed that a higher risk of MDR-TB among both new and previously treated cases was most strongly associated with a local increase in the percentage of TB cases that had previously been in detention (table 3). However, ROC analysis showed that the models had poor predictive probabilities (AUC 0.59 for each model) indicating that a substantial amount of the local heterogeneity in MDR-TB remained unexplained by individual-level characteristics that could be included in our models. 


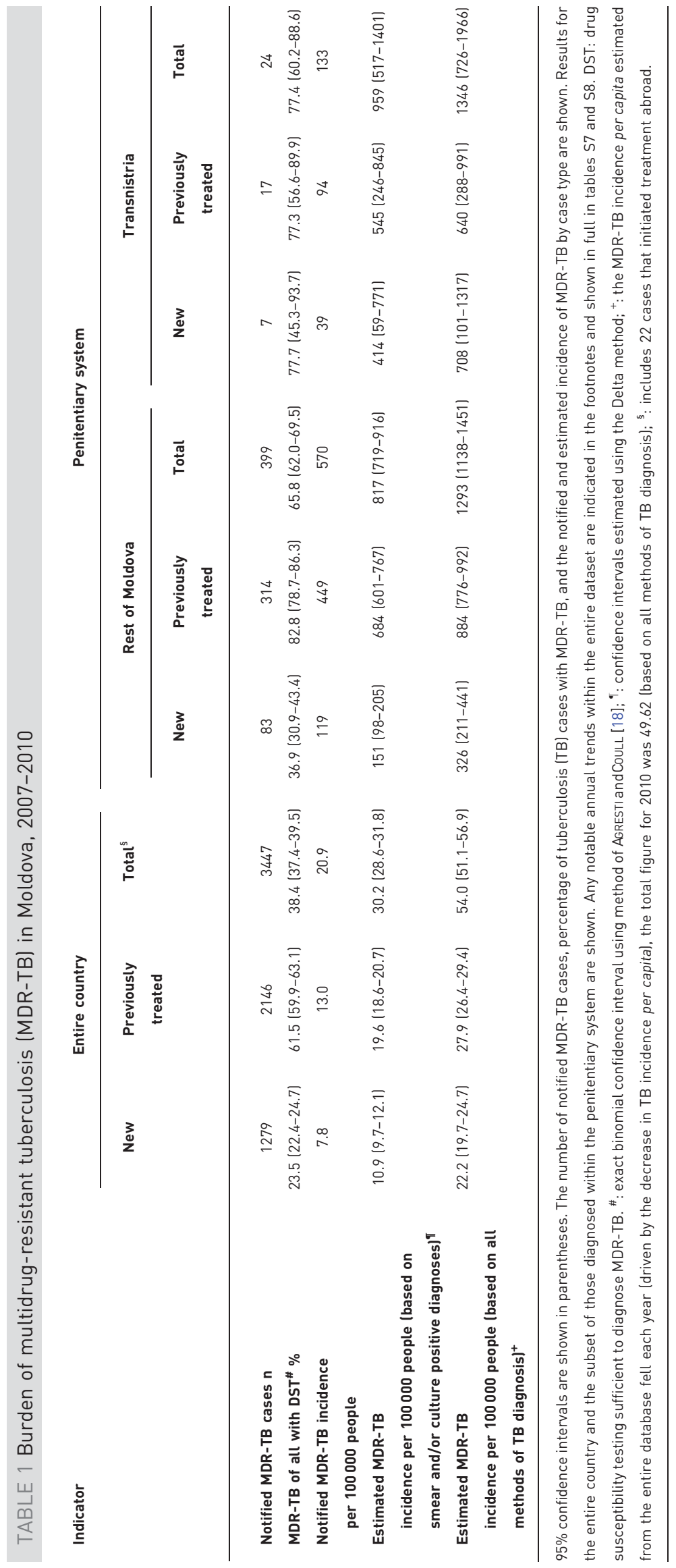




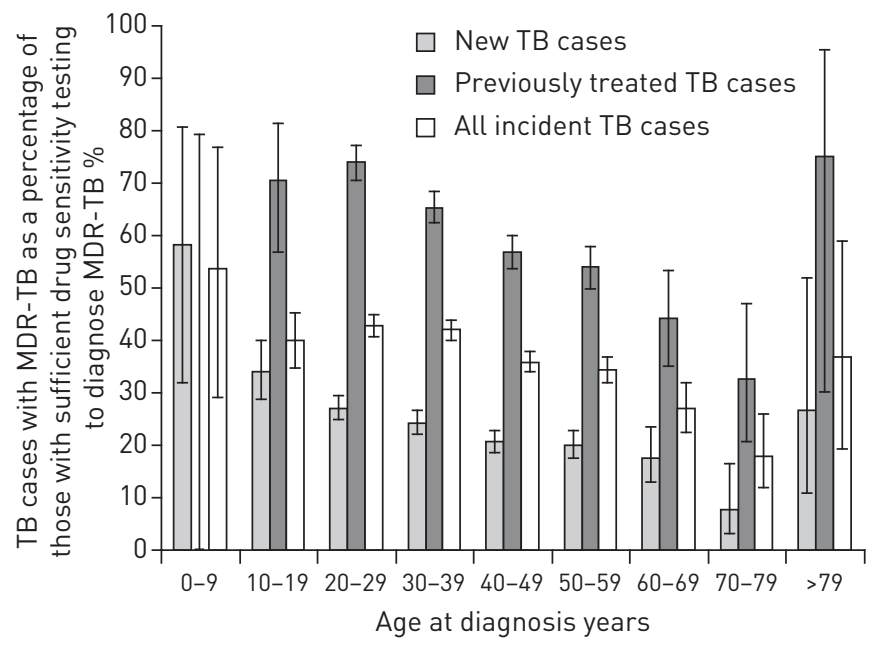

FIGURE 2 Number of tuberculosis (TB) cases confirmed to have multidrugresistant tuberculosis (MDR-TB) as a percentage of those with sufficient drug susceptibility testing to diagnose MDR-TB by age, 2007-2010. Binomial confidence intervals are shown.

\section{Discussion}

Our study demonstrates that the high MDR-TB burden shown by aggregate statistics in Moldova masks substantial spatial heterogeneity [19]. Identifying areas of high burden and risk of MDR-TB should help prioritise resources and allow for targeted studies aimed at understanding the local drivers of emergence and spread of MDR-TB [20]. We have found that the spatial variation in MDR-TB burden is striking; the estimated MDR-TB incidence rate in some rayons is 20 times that of others, and the percentage of new and

TABLE 2 Individual-level risk factors (i.e. those that are associated with an increased disease risk for an individual) for multidrug-resistant tuberculosis (MDR-TB) diagnosis in new and previously treated tuberculosis (TB) cases

\begin{tabular}{|c|c|c|c|c|c|}
\hline \multirow[t]{2}{*}{ Variable } & \multirow[t]{2}{*}{ Level } & \multicolumn{2}{|c|}{$\begin{array}{l}\text { Model for MDR-TB risk } \\
\text { among new TB cases }\end{array}$} & \multicolumn{2}{|c|}{$\begin{array}{l}\text { Model for MDR-TB risk among } \\
\text { previously treated TB cases }\end{array}$} \\
\hline & & $\begin{array}{l}\text { Change in odds of having } \\
\text { MDR-TB }(95 \% \mathrm{Cl})\end{array}$ & p-value ${ }^{\#}$ & $\begin{array}{l}\text { Change in odds of having } \\
\text { MDR-TB }(95 \% \mathrm{Cl})\end{array}$ & p-value $\#$ \\
\hline \multirow{2}{*}{$\begin{array}{l}\text { Age (linear) } \\
\text { TB contact in the } \\
\text { household }\end{array}$} & Each additional 10 years & $-10 \%(-7--14 \%)$ & $<0.0001$ & $-7 \%(-5--9 \%)$ & $<0.0001$ \\
\hline & $\begin{array}{l}\text { No } \\
\text { Yes }\end{array}$ & $\begin{array}{c}\text { Reference } \\
+59 \%(+37-+84 \% \text { increase })\end{array}$ & $<0.0001$ & & \\
\hline $\begin{array}{l}\text { In detention at the } \\
\text { time of diagnosis }\end{array}$ & Yes & Included as a confounder & 0.12 & $+8 \%(0-+17 \%)$ & 0.035 \\
\hline \multirow{2}{*}{$\begin{array}{l}\text { Was previously in } \\
\text { detention }\end{array}$} & No & Reference & & Reference & \\
\hline & Yes & $+38 \%(+19-+60 \%)$ & $<0.0001$ & $+27 \%(+20-+35 \%)$ & $<0.0001$ \\
\hline \multirow{2}{*}{$\begin{array}{l}\text { Living in an urban or } \\
\text { rural area }\end{array}$} & Rural & Reference & & Reference & \\
\hline & Urban & $+30 \%(+18-+44 \%)$ & $<0.0001$ & $+10 \%(+4-+16 \%)$ & 0.0003 \\
\hline $\begin{array}{l}\text { Was outside Moldova } \\
\text { for }>3 \text { months in } \\
\text { previous } 12 \text { months }\end{array}$ & No & Reference & & & \\
\hline \multirow[t]{2}{*}{ Homeless } & No & & & Reference & \\
\hline & Yes & & & $+9 \%(0-+19 \%)$ & 0.052 \\
\hline \multirow[t]{2}{*}{ Disabled } & No & & & Reference & \\
\hline & Yes & & & $+27 \%(+20-+36 \%)$ & $<0.0001$ \\
\hline Household size & & & & Included as a confounder & 0.14 \\
\hline
\end{tabular}

Cells left blank indicate that that variable was not included. " : from likelihood ratio test; " $89 \%$ of cases were HIV negative or positive, and only $11 \%$ were untested/tested with unknown result. 
a)

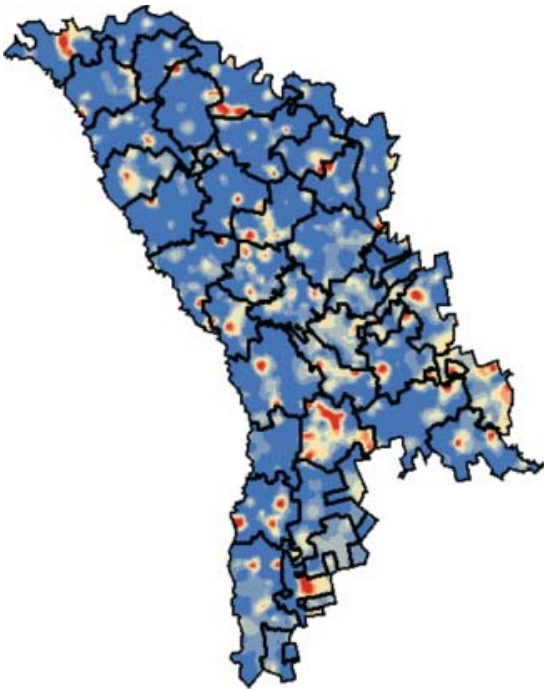

c)

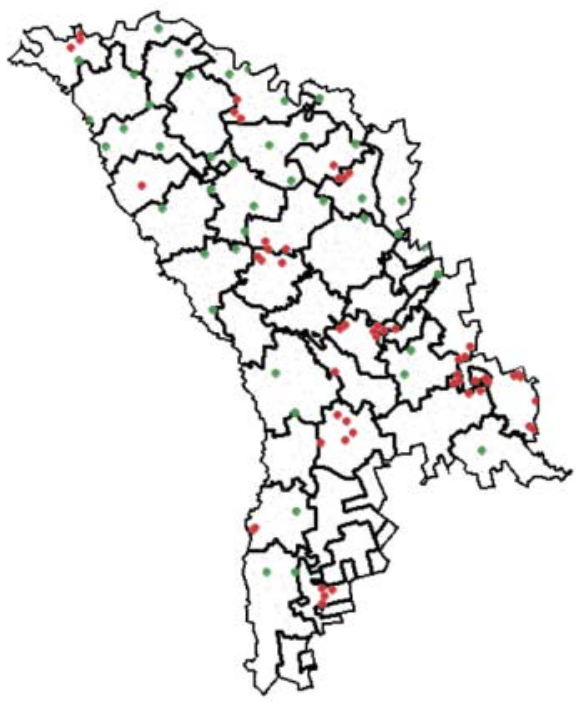

b)

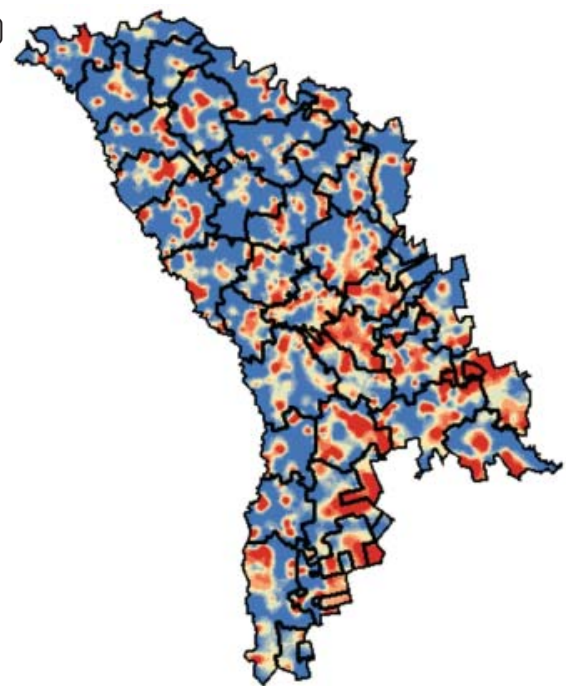

d)

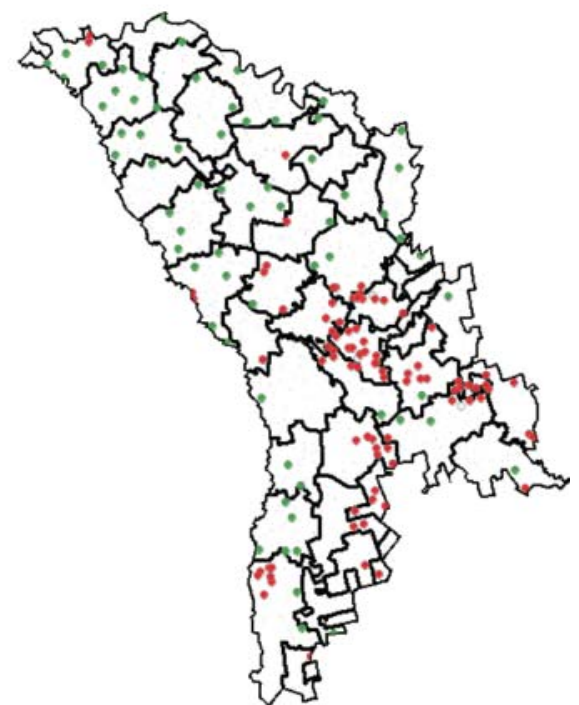

TB cases with MDR-TB:

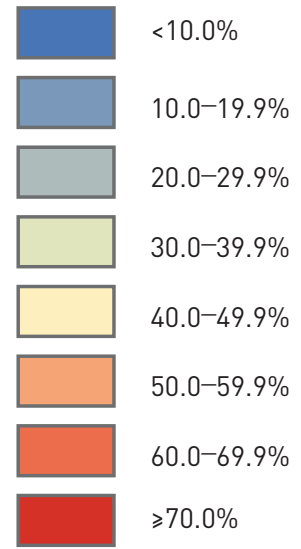

FIGURE 3 Maps of multidrug-resistant tuberculosis (MDR-TB) in the Republic of Moldova. Number of notified MDR-TB cases as a percentage of tuberculosis (TB) cases with drug susceptibility testing (DST) sufficient to diagnose MDR-TB among a) new TB cases and b) previously treated TB cases. c) and d) show spatial clustering analysis (using Local Moran's I) of data shown in a) and b), respectively, showing localities that are part of a statistically significant spatial cluster of high MDR-TB risk localities (red spots) or statistically significant outliers (i.e. localities with high MDR-TB next to areas of low MDR-TB risk, green spots). Maps shown in a) and b) were produced using data by locality, which was spatially smoothed using inverse distance weighting (see methods and online supplementary material methods section). New cases represent transmission of MDR-TB while previously treated cases include both acquired and transmitted MDR-TB infection. Areas that have a high percentage of TB cases with MDR-TB (fig. $3 a$ and $3 b$ ) may not necessarily have high MDR-TB incidence (fig. 4a and 4b), and thus figures $3 a$ and $3 b$ and figures $4 \mathrm{a}$ and $4 \mathrm{~b}$ have different interpretations. Locations in all panels are based on residential location and not location of diagnosis.

previously treated TB cases with MDR-TB ranges from $3 \%$ to $41 \%$ and $41 \%$ to $79 \%$, respectively. Reports from regional-level surveys in some countries indicate that this degree of spatial heterogeneity may be characteristic $[8,21]$ and suggests that micro-epidemics could be occurring, although more detailed molecular epidemiological studies are needed to confirm the underlying causes of these spatial patterns. Our study is the first to demonstrate this phenomenon across an entire country and to produce detailed maps showing that substantial heterogeneity may exist even within the boundaries of relatively small administrative regions (e.g. rayons).

That 23.5\% of new cases had MDR-TB indicates that transmission of MDR-TB has a substantial role in this epidemic. Young, urban residents are at particularly high risk of resistance, as are new TB cases with HIV co-infection. While the association between HIV and MDR-TB varies by setting $[22,23]$, this result concurs with univariable analyses from other FSU countries $[8,21,24]$. Our analysis is the first to adjust for multiple potential confounders providing robust evidence for this association, at least in this setting. 
a)

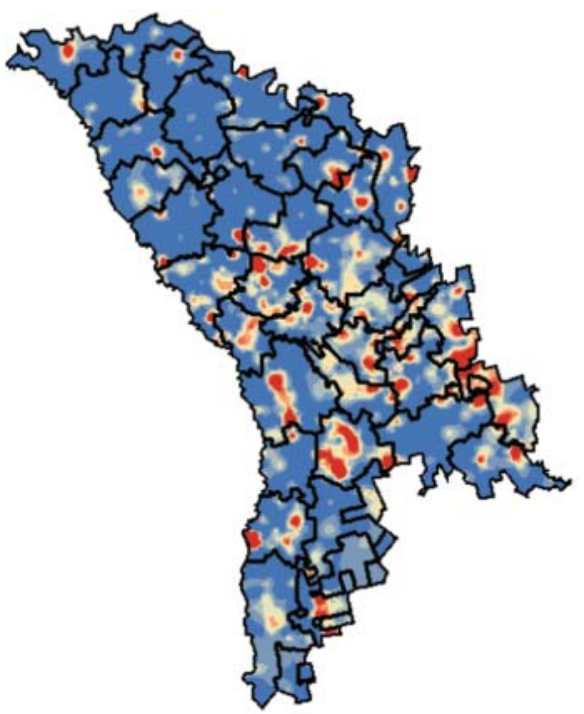

c)

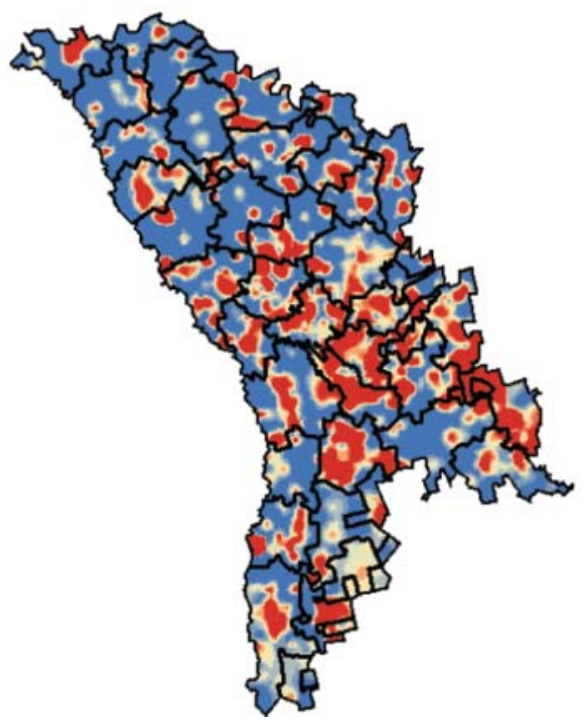

b)

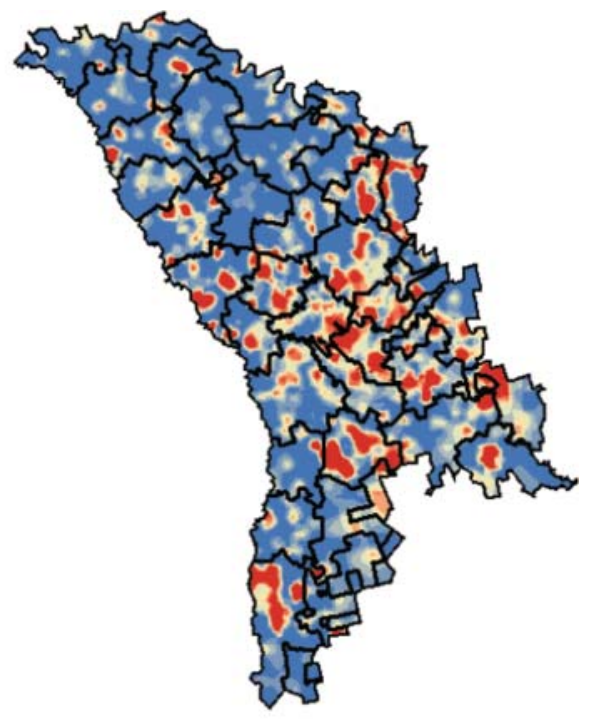

d)

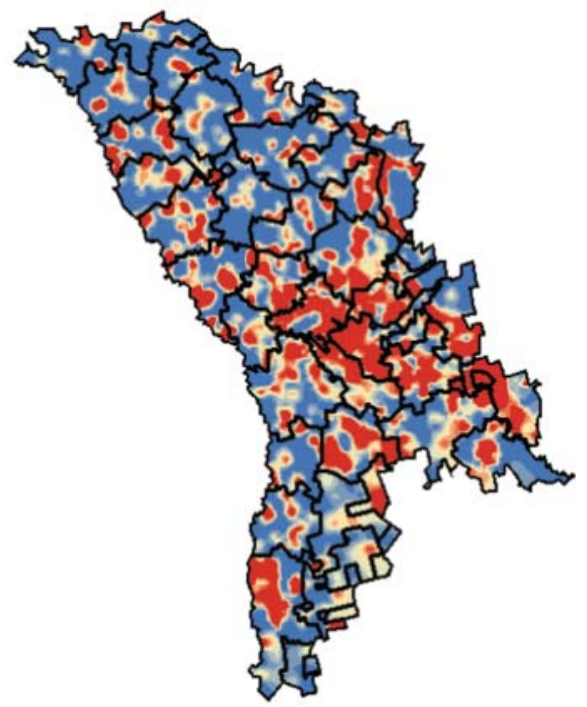

MDR-TB incidence per 100000 people per year:

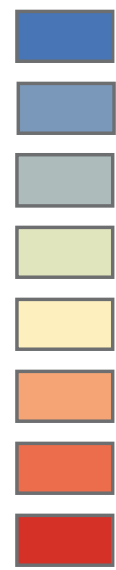

$<4.0$

4.0-7.9

$8.0-11.9$

$12.0-15.9$

$16.0-19.9$

$20.0-23.9$

$24.0-27.9$

$\geqslant 28.0$

FIGURE 4 Annual notified multidrug-resistant tuberculosis (MDR-TB) incidence per 100000 population among a) new tuberculosis (TB) cases and b) previously treated TB cases and annual estimated MDR-TB incidence per 100000 population among c) new TB cases and d) previously treated cases. All maps were produced using data by locality that was spatially smoothed using inverse distance weighting (see methods section and online supplementary material methods section). New cases represent transmission of MDR-TB while previously treated cases include both acquired and transmitted MDR-TB infection. Areas which have a high percentage of TB cases with MDR-TB (fig. 3a and 3b) may not necessarily have high MDR-TB incidence and thus figure 3a and 3b and figure $4 \mathrm{a}$ and $4 \mathrm{~b}$ have different interpretations. Estimated incidence (fig. $4 \mathrm{c}$ and $4 \mathrm{~d}$ ) assumes that the percentages shown in figures $3 \mathrm{a}$ and $3 \mathrm{~b}$ are applicable to all TB cases (including those without positive culture and drug susceptibility testing results). Locations in all panels are based on residential location and not location of diagnosis.

Two-thirds of all TB cases diagnosed in Moldovan prisons had MDR-TB and the estimated annual incidence was at least 570 (possibly as high as 1300) per 100000 people. We believe that this is the highest estimated incidence of MDR-TB ever reported. While it is unsurprising that MDR-TB rates are higher in prisons $[7,25]$, the percentages with MDR-TB are greater than those from other studies among prisoners in the FSU $[24,26]$. While improvements have been made within Moldovan prisons, case detection and the use of TB diagnostics appear less accessible within the Transnistrian penitentiary system, suggesting that there may be a substantial burden of undiagnosed drug resistance.

We found that a higher local risk of MDR-TB was associated with an increase in the percentage of TB cases that had previously been in detention; this suggests that the high rates of MDR-TB in prisons may be 
TABLE 3 Locality-level risk factors for multi-drug resistant tuberculosis (MDR-TB) diagnosis in new and previously treated tuberculosis (TB) cases

Percentage of TB cases in the locality that
Model for MDR-TB risk

among new TB cases

\begin{tabular}{|c|c|c|c|c|}
\hline & & \\
\hline & $\begin{array}{l}\text { Change in odds of a TB case } \\
\text { having MDR-TB }(95 \% \mathrm{CI})\end{array}$ & p-value & $\begin{array}{l}\text { Change in odds of a TB case } \\
\text { having MDR-TB }(95 \% \mathrm{Cl})\end{array}$ & p-value \\
\hline Are aged 0-39 years old & $+3.3 \%(-0.7-+7.4 \%)$ & 0.10 & $+6.4 \%(+2.1-+10.9 \%)$ & 0.003 \\
\hline Have a TB contact in the household & $+0.5 \%(-2.0-+1.1 \%)$ & 0.57 & & \\
\hline Are currently in detention & & & $-0.9 \%(-3.0-+1.3 \%)$ & 0.40 \\
\hline Have previously been in detention & $+4.7 \%(+2.8-+6.7 \%)$ & $<0.0001$ & $+6.7 \%(+4.4-+9.0 \%)$ & $<0.0001$ \\
\hline Live in an urban area & $+0.1 \%(-0.7-+0.8 \%)$ & 0.85 & $-1.4 \%(-2.2--0.5 \%)$ & 0.002 \\
\hline $\begin{array}{l}\text { Were outside Moldova for }>3 \text { months } \\
\text { in the previous } 12 \text { months }\end{array}$ & $-4.0 \%(-5.6--2.3 \%)$ & $<0.0001$ & & \\
\hline Are HIV positive & $0.0 \%(-1.3-+1.3 \%)$ & $>0.99$ & & \\
\hline Are homeless & & & $-2.1 \%(-3.8--0.5 \%)$ & 0.011 \\
\hline Are disabled & & & $+0.8 \%(-1.2 \%-+2.9 \%)$ & 0.43 \\
\hline
\end{tabular}

Model for MDR-TB risk among previously treated TB cases

Cells left blank indicate that that variable was not included. Changes in odds of MDR-TB (among TB cases) are shown for each $20 \%$ proportionate increase in the explanatory variable (e.g. from $50 \%$ to $60 \%$ ).

$\begin{array}{lc}+3.3 \%(-0.7-+7.4 \%) & 0.10 \\ +0.5 \%(-2.0-+1.1 \%) & 0.57 \\ +4.7 \%(+2.8-+6.7 \%) & <0.0001 \\ +0.1 \%(-0.7-+0.8 \%) & 0.85 \\ -4.0 \%(-5.6--2.3 \%) & <0.0001 \\ & >0.99\end{array}$

$-2.1 \%(-3.8--0.5 \%)$

disproportionately contributing to increased local rates in the civilian sector. This could occur if prisoners become infected with MDR-TB whilst in detention, develop active disease following release and subsequently spread these highly resistant strains in the community [27]. Our finding that cases who had previously been detained were at an increased risk of MDR-TB also supports this potential explanation. Previous studies have concluded that prisons may have a substantial role in fuelling TB epidemics at the population level (i.e. across a geographic area and not just for an individual case) [25, 28], and our study provides additional data to support this theory.

A strength of our study is the high percentage of culture-positive TB cases receiving quality-assured DST ( $96 \%$ by 2010). High coverage with diagnostics and a detailed patient database have allowed us to document the MDR-TB situation in Moldova with high resolution. Given similar historical approaches for TB control used in other FSU countries, the insights generated in Moldova may contribute to improved understanding of the resistance in this global region where MDR-TB is most concerning.

An important limitation of our study is our inability to fully assess the contribution that nosocomial transmission may have to MDR-TB incidence. Similar to many settings in the region, all TB patients in Moldova initiate treatment within hospitals. Additional molecular epidemiological studies within these settings $[21,29]$ are needed and will have implications for both infection control within hospitals and the use of ambulatory treatment.

Our study is also limited by the programmatic nature of the data and the reliance on existing approaches for TB case detection. It is possible that the quality of TB case detection varies by location and could partially explain the observed heterogeneity in MDR-TB risk. However, we consider this unlikely to have had a substantial impact as Moldova is a small country with little geographical variation in terrain and welldistributed points to access TB care. Only about half of all notified pulmonary TB cases had positive cultures. Possible explanations for relatively low culture positivity rates include sub-optimal specimen collection, transport, or handling or incomplete entry of updated laboratory results into the database. Our estimates within the Transnistrian penitentiary system are especially prone to bias due to the relatively poor utilisation of culture and DST.

While our locality-level model helped us to identify the presence of higher proportions of previously detained individuals as potential contributors to higher local MDR-TB risk, the model did not explain a large percentage of the local variability in resistance. This demonstrates that currently available data are not sufficient to explain local patterns in MDR-TB and further studies are needed to provide insight into why specific areas bear relatively high burden and risk. Nonetheless, the spatial maps produced here provide information that can inform at least two types of action. First, identifying hotspots of burden and risk of MDR-TB permits efficient deployment of interventions that are often in limited supply to areas where they 
are most urgently needed. Secondly, these hotspots serve as ideal places to locate studies to identify local causes of resistance and to trial novel interventions.

MDR-TB presents a serious threat to public health in many global settings; to date the scale of response to highly drug-resistant disease has failed to meet the actual need [4,30]. This study underscores the role that comprehensive surveillance and spatial analysis of MDR-TB can play in understanding the local epidemiology of MDR-TB and improving responses to this crisis.

\section{Acknowledgements}

We thank all those involved in surveillance, laboratory testing and treatment for TB in the Republic of Moldova.

The content is solely the responsibility of the authors and does not necessarily represent the official views of the National Institute of General Medical Sciences or the National Institutes of Health. The funders had no role in study design, data collection and analysis, decision to publish, or preparation of the manuscript. M. Zignol, A. Dadu and M. Dara are staff members of the World Health Organization (WHO). The authors alone are responsible for the views expressed in this publication and they do not necessarily represent the decisions or policies of WHO.

\section{References}

1 World Health Organization. Global Tuberculosis Control 2011. Geneva, 2011. Report number WHO/HTM/TB/ 2011.16. www.who.int/tb/publications/global_report/2011/gtbr11_full.pdf Date last accessed: January 23, 2013.

2 Gandhi NR, Nunn P, Dheda K, et al. Multidrug-resistant and extensively drug-resistant tuberculosis: a threat to global control of tuberculosis. Lancet 2010; 375: 1830-1843.

3 Espinal MA, Kim SJ, Suarez PG, et al. Standard short-course chemotherapy for drug-resistant tuberculosis: treatment outcomes in 6 countries. JAMA 2000; 283: 2537-2545.

4 Zignol M, van Gemert W, Falzon D, et al. Surveillance of anti-tuberculosis drug resistance in the world: an updated analysis, 2007-2010. Bull World Health Organ 2012; 90: 111-119.

5 Skrahina A, Hurevich H, Zalutskaya A, et al. Alarming levels of drug-resistant tuberculosis in Belarus: results of a survey in Minsk. Eur Respir J 2012; 39: 1425-1431.

6 World Health Organization. Towards universal access to diagnosis and treatment of multidrug-resistant and extensively drug-resistant tuberculosis by 2015: WHO progress report 2011. Geneva, 2011. Report number WHO/ HTM/TB/2011.3. http://whqlibdoc.who.int/publications/2011/9789241501330_eng.pdf Date last accessed: January 23, 2012.

7 Dara M, Kluge H. Roadmap to prevent and combat drug-resistant tuberculosis. Copenhagen, WHO Regional Office for Europe, 2011. www.euro.who.int/_data/assets/pdf_file/0014/152015/e95786.pdf Date last accessed: January 23,2013

8 World Health Organization. Multidrug and extensively drug-resistant (M/XDR-TB). 2010 Global Report on Surveillance and Response. Geneva, 2010. Report number WHO/HTM/TB/2010.3 http://whqlibdoc.who.int/ publications/2010/9789241599191_eng.pdf Date last accessed: January 23, 2013.

9 International Organization for Migration. Migration in Moldova: A Country Profile 2008. Geneva, 2008. http://publications.iom.int/bookstore/free/Moldova_Profile2008.pdf Date last accessed: January 23, 2013.

10 Crudu V. Anti-tuberculosis drug resistance surveillance, Republic of Moldova, 2006. Chisinau, 2009. Available from valeriu.crudu@gmail.com

11 Soltan V, Henry AK, Crudu V, et al. Increasing tuberculosis case detection: lessons from the Republic of Moldova. Bull World Health Organ 2008; 86: 71-76.

12 Population Census Results. 2004. www.statistica.md/pageview.php?1=en\&idc=295 Date last accessed: January 23, 2013.

13 World Health Organization. Guidelines for drug resistance surveillance in tuberculosis, 4th Edn. Geneva, 2009. Report number WHO/HTM/TB/2009.422 http://whqlibdoc.who.int/publications/2009/97892415986675_eng.pdf Date last accessed: January 23, 2013.

14 Shepard D. A two-dimensional interpolation function for irregularly-space data. In: 23rd Association for Computing Machinery National Conference, 1968. New York, Association for Computing Machinery, 1968; pp. 517-524.

15 Anselin L. Local Indicators of Spatial Association - LISA. Geograph Anal 1995; 27: 93-115.

16 Cox D. Some statistical methods connected with series events. J Royal Stat Soc Series B Met 1955; 17: 129-164.

17 Brooker S, Hay SI, Bundy DA. Tools from ecology: useful for evaluating infection risk models? Trends Parasitol 2002; 18: 70-74.

18 Agresti A, Coull BA. Approximate is better than "exact" for interval estimation of binomial proportions. Am Statistician 1998; 52: 119-126.

19 Cohen T, Colijn C, Wright A, et al. Challenges in estimating the total burden of drug-resistant tuberculosis. Am J Respir Crit Care Med 2008; 177: 1302-1306.

20 Cohen T, Manjourides J, Hedt-Gauthier B. Linking surveillance with action against drug-resistant tuberculosis. Am J Respir Crit Care Med 2012; 186: 399-401.

21 Wright A, Zignol M, Van Deun A, et al. Epidemiology of antituberculosis drug resistance 2002-07: an updated analysis of the Global Project on Anti-Tuberculosis Drug Resistance Surveillance. Lancet 2009; 373: 1861-1873.

22 Wells CD, Cegielski JP, Nelson LJ, et al. HIV infection and multidrug-resistant tuberculosis: the perfect storm. J Infect Dis 2007; 196: S86-S107.

23 Suchindran S, Brouwer ES, Van Rie A. Is HIV infection a risk factor for multi-drug resistant tuberculosis? A systematic review. PLoS One 2009; 4: e5561.

24 Dubrovina I, Miskinis K, Lyepshina S, et al. Drug-resistant tuberculosis and HIV in Ukraine: a threatening convergence of two epidemics? Int J Tuberc Lung Dis 2008; 12: 756-762.

25 Stuckler D, Basu S, McKee M, et al. Mass incarceration can explain population increases in TB and multidrugresistant TB in European and central Asian countries. Proc Natl Acad Sci USA 2008; 105: 13280-13285. 
Ruddy M, Balabanova Y, Graham C, et al. Rates of drug resistance and risk factor analysis in civilian and prison patients with tuberculosis in Samara Region, Russia. Thorax 2005; 60: 130-135.

27 Reyes H, Coninx R. Pitfalls of tuberculosis programmes in prisons. BMJ 1997; 315: 1447-1450.

28 Basu S, Stuckler D, McKee M. Addressing institutional amplifiers in the dynamics and control of tuberculosis epidemics. Am J Trop Med Hyg 2011; 84: 30-37.

29 Nodieva A, Jansone I, Broka L, et al. Recent nosocomial transmission and genotypes of multidrug-resistant Mycobacterium tuberculosis. Int J Tuberc Lung Dis 2010; 14: 427-433.

30 Keshavjee S, Farmer PE. Picking up the pace - scale-up of MDR tuberculosis treatment programs. N Engl J Med 2010; 363: 1781-1784. 